\title{
Doppler Ultrasound Velocity Mapping of Extra-ocular Muscles: A Preliminary Report
}

\author{
C. R. CANNING and M. RESTORI \\ London
}

\begin{abstract}
Summary
Colour doppler imaging and conventional spectral doppler/B-mode techniques were used to detect and quantify rectus muscle movement during voluntary saccades. A velocity gradient was evident in all muscles studied-low velocities near the muscle origin at the back of the orbit increasing linearly to a maximum near the muscle insertion on the globe. A consistent reduction in velocity occurred along muscles injected with botulinum toxin. Doppler ultrasound complements current clinical eye movement recording techniques_electronystagmography, infrared light reflection methods and magnetic induction methods-insofar as it detects movement in parts of the muscle itself rather than movement of the globe secondary to muscle contraction. Future developments in colour doppler imaging will make the technique clinically more applicable.
\end{abstract}

Many techniques have been developed to measure eye movements objectively. They have found application in research into eye movement disorders as well as the clinical evaluation of selected patients. All tests currently in use measure movement of the eye and infer extra-ocular muscle movement from this. Ultrasonically the movement of the extra-ocular muscles can be imaged using the real-time $\mathrm{B}$-mode technique, but quantitative measurements of muscle velocity using this technique are tedious. Doppler ultrasound techniques on the other hand, which are based on measurement of the frequency change induced when a transmitted pulse is scattered by a moving target, can also be used to measure muscle velocities. Recent technical advances have allowed the simultaneous display of a B-mode image and doppler frequency shift information on the same screen. Application of these techniques to the eye muscles offers the possibility of directly measuring muscle movement.

This paper presents some preliminary results and discusses the limitations of the method using current doppler ultrasound technology.

\section{Techniques}

An Acuson 128 system, capable of operating simultaneously in a combination of pulsed doppler mode and B-mode, was used in this study. A 5 megahertz probe, comprising 128 transducer elements, was coupled to the closed eyelid of a seated patient via liquid gel.

Doppler techniques are based on the measurement of the frequency change (the doppler shift frequency) induced when a transmitted ultrasonic wave of frquency $f_{i}$ is scattered by moving tissue. The doppler shift equation is given below for an idealised single scatterer moving with a constant velocity $\mathrm{V}$ at 


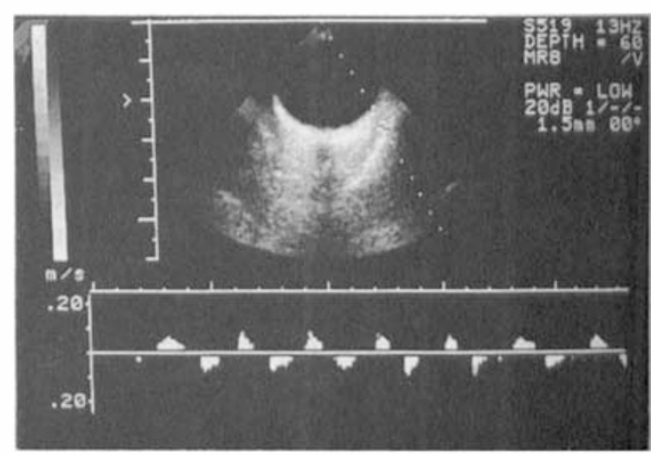

Fig. 1. Colour doppler image taken during a saccade. Moving tissues are highlighted in colour (reproduced here in black and white) and superimposed on the $B$-mode image. The muscle (see cursor positioned centrally within it) is not well resolved due to movements of adjacent tissues. A velocity gradient is not apparent as velocities are concentrated at the low end of the colour scale.

an angle of $\theta$ to the incident ultrasonic wave. It demonstrates that returned echoes have a higher frequency than transmitted pulses if the scatterer is moving towards the transducer, and a lower frequency if the scatterer is moving away from a transducer; the magnitude of the doppler shift frequency is proportional to the velocity of the scatterer.

Equation $^{1} \ldots \mathrm{f}_{\mathrm{D}}=\mathrm{f}_{\mathrm{i}}-\mathrm{f}_{\mathrm{r}}=\frac{2 \mathrm{v} \cdot \mathrm{f}_{\mathrm{i}} \cos \theta}{\mathrm{c}}$

where:

$$
\begin{aligned}
f_{D}= & \text { doppler shift frequency } \\
f_{i}= & \text { incident frequency of transmitted } \\
& \text { wave } \\
f_{r}= & \text { returned frequency of echoes } \\
\mathrm{V}= & \text { velocity of scatterer } \\
\theta= & \text { angle between the direction of travel } \\
& \text { of transmitted ultrasonic wave and } \\
& \text { direction of scatterer flow } \\
c= & \text { sound velocity in the medium sur- } \\
& \text { rounding scatterer }
\end{aligned}
$$

\section{Colour Doppler Imaging}

The same transducer elements are used to collect information for both B-mode and doppler imaging. The amplitude and time delay of returned echoes are used to form the greyscale B-mode image, and colour encoded doppler shift frequency information from all or part of the scan area is superimposed upon this; the combined image is usually referred to as the colour doppler image. Generally shades of red and blue are used to represent different tissue velocities towards or away from the transducer elements; stationary tissues appear in shades of grey (Fig. 1). A compromise must be made between the resolution of the colour image and the colour doppler imaging frame rate. The resolution of the colour encoded doppler shift information is less than the spot size of the grey-scale B-mode image. A frame rate of 19 colour doppler images per second is achieved. The technique has been applied primarily to imaging blood flow, so the colour ranges mostly cover those velocities usually encountered in blood flow studies.

\section{Combined Spectral Doppler and B-Mode Imaging Techniques}

An alternative way of displaying doppler information is the spectral doppler method. A region for study is selected on the B-mode image by bracketing it using a small gate displayed as two horizontal lines (Fig. 2). A graph of doppler shift frequency versus time-the spectrogram-may then be presented directly beneath the B-mode image. The brightness of any point on this graph is a

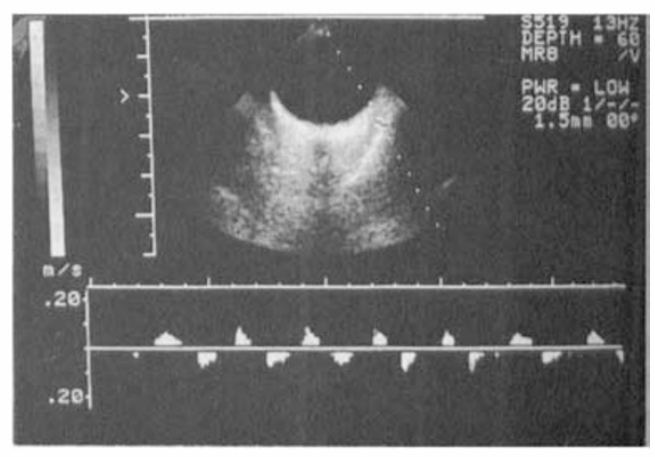

Fig. 2. Top: B-mode image of the eye and orbit; the dashed line represents the direction of travel of the ultrasonic pulses and the two horizontal bars represent a gate positioned in the lateral rectus muscle within which doppler signals are analysed.

Bottom: spectrogram derived from the gated region of muscle shown above. The spectrogram is a graph of doppler shift frequency versus time with display brightness representing the number of scatterers exhibiting a particular doppler frequency shift. Points above the $X$-axis represent movement towards a transducer and those beneath movement away from a transducer. Time scale five seconds. 
measure of the number of scatterers within the sample volume exhibiting a particular doppler shift frequency. Traditionally, movement towards a transducer is plotted above the $\mathrm{X}$-axis and movement away from a transducer beneath the $\mathrm{X}$-axis. As the doppler shift frequency is proportional to the component of tissue velocity directly towards or away from the probe, the Y-axis is calibrated in metres per second. Typically a time axis scale on the spectrogram of five seconds per sweep is selected. Collection and analysis of the spectral doppler data allows simultaneous B-mode imaging frame rates of 14 per second. Any change in a spectrogram due to a change in the sample volume is thus immediately apparent on the B-mode image.

\section{Muscle Studies}

Using the colour doppler imaging mode, the extraocular muscles to be studied were identified and the subject was then asked to gener-

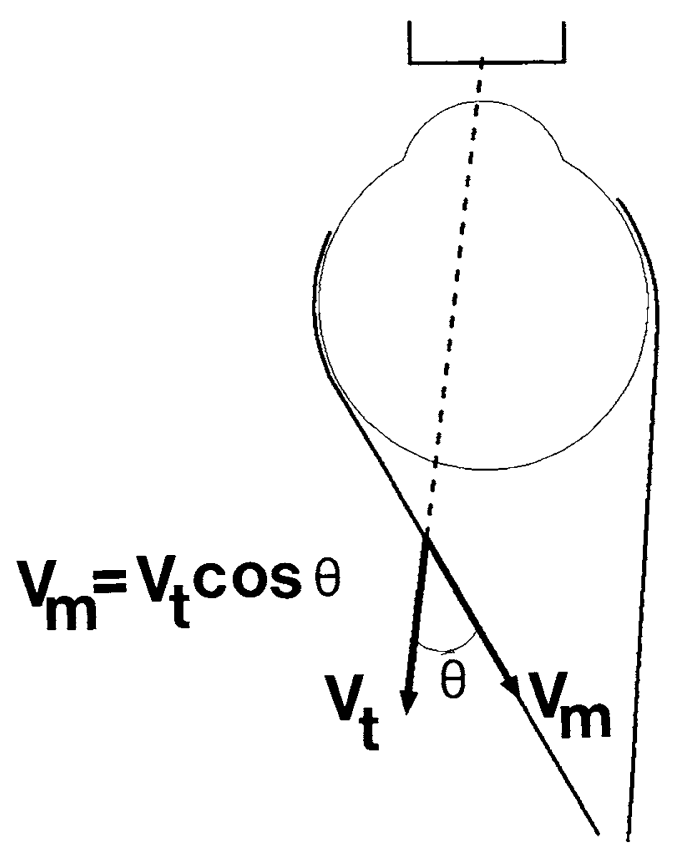

Fig. 3. Diagram to show the angle $\theta$ between the direction of travel of the ultrasonic pulses and the line of action of the muscle. Angle $\theta$ will vary along the length of the muscle. The velocity of the muscle along its line of action Vm, can be calculated using its component with respect to the transducer $V t$, and angle $\theta$ from Equation 1. ate large saccadic eye movements in the direction of action of those muscles. The extent of the saccadic excursion (50 degrees) was controlled by keeping open the eye not being examined. The most sensitive colour range was selected and the saccades were recorded on video tape for later analysis.

Using the combined doppler spectrogram and B-mode, a single extra-ocular muscle (usually the lateral rectus) was identified. Muscles appear as dark bands within the more echogenic orbital fat. The doppler gate was positioned within it close to the globe. The subject again generated saccades toward and away from the direction of action of the muscle and the doppler spectrogram was printed (Fig. 2). The gate was then moved to a different position within the muscle and the saccades repeated. The spectrograms from three or four such positions were examined. Peak velocity along the axis of the transducer during each saccade was measured and averaged for each position in the muscle. This was converted to velocity along the axis of the muscle by dividing by the cosine of the angle $\theta$; the angle of $\theta$ was measured on the B-mode image (Fig. 3).

Ten normal eyes were studied. In addition, four eyes in which botulinum toxin had been injected into either the medial or lateral rectus muscles were studied one week after the injection. The toxin was injected into the antagonist of paretic horizontal rectus muscles in these cases to reduce the angle of squint. Toxin weakens the injected muscle.

\section{Results}

Colour doppler imaging showed muscle movement quite clearly as a blush of blue on one side of the globe and orbit, and red on the other. These represented movement away from and toward the probe respectively. The technique was unable to discriminate differences in muscle velocity along the length of the muscles even on the most sensitive colour range. In addition, movement of the muscle was accompanied by movement of adjacent tissue septa and fat from which the muscle itself could not reliably be distinguished.

Doppler spectrography is able to measure low velocities more precisely. Moreover, the extraocular muscles appear quite clearly on 


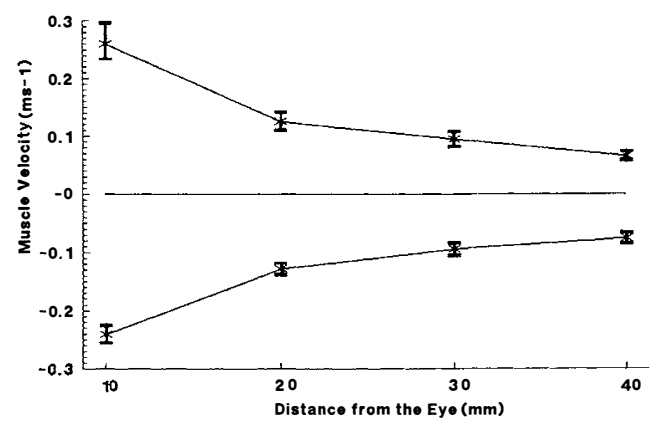

Fig. 4 Results of multiple measurements of a medial (positive velocities) and lateral (negative velocities) rectus muscle in the same eye show little variation in saccadic velocity at a given point in the muscle (error bars are \pm 1 standard deviation). A velocity gradient is apparent along the length of each muscle.

the B-mode image as dark stripes surrounded by more echogenic fat. Considerable variation in saccadic velocities of the ten normal eyes was present, but not between repeated saccades of the same eye (Fig. 4). When studying the lateral rectus, movement toward the transducer is due to medial rectus contraction with lateral rectus relaxation, and vice versa. Muscle velocity was always least at the back of the orbit, and maximal close to the globe. Velocities toward and away from the transducer were not significantly different.

Botulinum toxin had been injected into the lateral rectus of three eyes and the medial rectus of one eye. Movement in the direction of action of the treated muscle was slowed compared to its antagonist, even though the antagonist was already paretic (Fig. 5).

The velocity gradients of the normal and botulinum treated eyes were compared by normalising the data (Fig. 6). No significant differences were apparent between these gradients.

\section{Discussion}

Many disorders of the oculomotor system are characterised by specific patterns of eye movement, and objective recording of such patterns has contributed to an understanding of the aetiology of the disorders.

Since the introduction of the forced duction test by Wolff in $1900,{ }^{1}$ many eye movement recording techniques have been developed. Quantification of the forced duction test was achieved by a variety of springs ${ }^{2}$ and strain gauges. ${ }^{3-6}$ Another approach measured the force generated by a muscle during active, voluntary duction using a modified Weck forceps as a torque gauge. ${ }^{7}$ These tests were invasive and, when done in the out-patient department, required a degree of co-operation from the patient which limited their applicability in children. A test for restrictive eye movement, which compared the intraocular pressure in the primary position with that in the affected direction of gaze, needed less co-operation from the patient but had limited sensitivity and specificity. ${ }^{8}$

Electronystagmography (ENG) —or electrooculography (EOG)-measures the alteration of the cornea-retina electrical potential difference induced by eye movement, and has been a useful clinical tool for years. It is noninvasive, measures both eyes together, and is sensitive to movements of one to two degrees over a range of 70-80 degrees horizontally and 40-50 degrees vertically. ${ }^{9}$ The resolution is limited by superimposed electromagnetic interference, electromyographic signals, lowfrequency drift and eyelid artefacts, the latter particularly affecting vertical eye movements.

Infrared light reflection off the cornea, pupil or corneo-scleral junction gives information about eye position with respect to the head. The system may be sensitive to much smaller eye movements (three minutes of

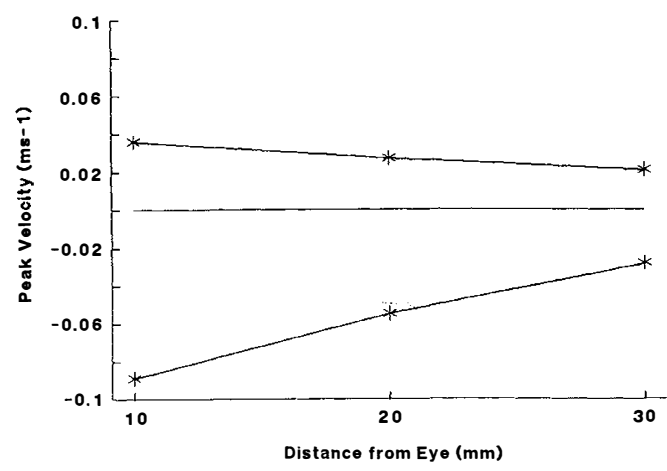

Fig. 5. The medial rectus muscle had been injected with botulinum toxin one week earlier and shows marked reduction in velocities throughout its length (above the line). The saccadic velocity of the antagonist lateral rectus (below the line) was also reduced, compared with normal muscles, because of abducent nerve palsy. 

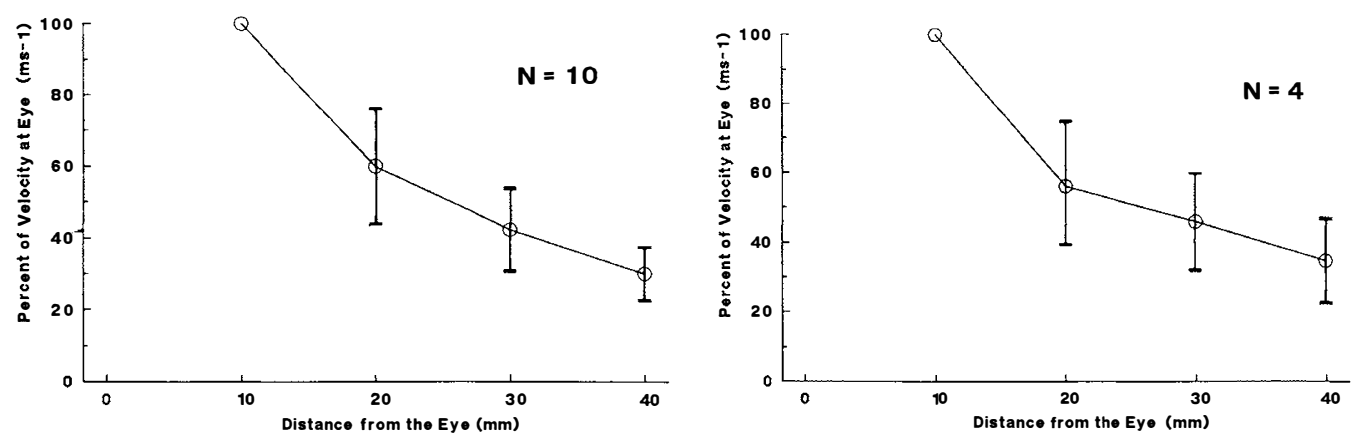

Fig. 6. Normalised data from (a) normal and (b) botulinum toxin treated muscles. There is no signficant difference between the slopes of the velocity gradients in these two groups.

arc), is non-invasive and can measure both eyes simultaneously. ${ }^{9}$ Recording vertical eye movements may be less accurate, because the corneo-scleral junction is covered by the eyelids above and below.

Magnetic induction methods work by inducing a varying magnetic field in a coil applied to the eye in a contact lens. ${ }^{10}$ Measuring the alterations in the field caused by eye movement allows accurate measurement of vertical, horizontal and torsional movement. The technique is more invasive since physical contact with the eye is required and usually only measures one eye at a time.

All these clinical eye movement recording methods document movement of the eye in response to movement of the extra-ocular muscles. Doppler ultrasound offers the possibility of measuring the actual muscle movement.

Current colour doppler imaging systems are designed primarily to measure blood flow rate in large blood vessels. As a result, the colour range provided by the machines cover the typical velocities found in such vessels. Saccadic eye movement velocity depends on the size of the saccades, ranging from 30 degrees per second for small movements up to 700 degrees per second for large ones. ${ }^{11}$ This equates to velocities within the muscle close to the globe in the range of 0.01 to $0.15 \mathrm{~ms}^{-1}$ These are at the low end of the colour doppler velocity scale which partly explains why velocity gradients were not demonstrated along the length of the muscles using this technique.

The doppler spectrogram method is more sensitive to lower velocities. It does, however, require measurements to be made sequentially along the length of the muscle using different saccades rather than simultaneously using the same saccade. Whilst the results suggest that variations between saccades are small (Fig. 4), this is a potential source of error.

At the orbital apex, movement of the optic nerve may cause a portion of it to be included within the doppler gate, so the recorded spectrogram is no longer entirely derived from muscle movement.

Doppler ultrasound techniques measure muscle velocity in one eye at a time. The equipment needed is expensive. It does, however, measure linearly over the whole range of the muscle movement and is non-invasive. Its overriding advantage is its ability to record movement actually within parts of the muscle rather than relying on the secondary movement of the globe. Doppler spectrogram analysis takes too long for this to be a useful clinical tool. Colour doppler imaging systems are being developed which respond to lower velocities. If they fulfil their promise and do allow complete analysis of muscle movement from a single saccade, then several avenues of clinical application could be explored such as in the assessment of orbital floor fractures, Brown's syndrome, Duane's syndrome, dysthyroid ophthalmopathy, myasthenia gravis, chronic progressive ophthalmoplegia, and muscles lost at surgery. Combining doppler ultrasound with simultaneous electromyographic recording may give infor- 
mation on the latency times and propagation of muscle contraction.

The authors wish to thank Acuson Ltd for technical support and Mr. P Fells for his helpful comments and encouragement. Mr. Canning was a Friends of Moorfields Research Fellow.

\section{References}

1 Wolff J: The occurrence of retraction movements of the eyeball together with congenital defects of the external ocular muscles. Arch Ophthalmol 1900, 29: 297-309.

${ }^{2}$ Schillinger RJ: The prevention of over-correction and under-correction in horizontal strabismus surgery. J Paediatr Ophthalmol 1966, 3: 38-41.

${ }^{3}$ Kaneko H, Koga A, Adachi K: Forced duction test. Acta Soc Ophthalmol Jpn 1971, 75: 1515-23.

${ }^{4}$ Metz HS and Cohen G: Quantitative forced traction test in strabismus. In Reinecke RD (ed). Proceedings of the fourth meeting of the International Strabismological Association, 1982 Asilomar CA. New York: Grune \& Stratton, 1984, 755-66.
${ }^{5}$ Rosenbaum AL and Myer JH: New instrument for the quantitative determination of passive forced traction. Ophthalmology 1980, 87: 158-63.

${ }^{6}$ Metz HS and Cohen GH: Quantitative forced duction measurements. Am Orthop J 1984, 34: 28-38.

${ }^{7}$ Scott AB, Collins CC, O'Meara DM: A forceps to measure strabismus forces. Arch Ophthalmol 1972, 88: $330-3$.

${ }^{8}$ Zappia RJ, Winkelman JZ, Gay AJ: Intraocular pressure changes in normal subjects and the adhesive muscle syndrome. Am J Ophthalmol 1971, 71: $880-3$.

${ }^{9}$ Reulan JPH: Fundamentals and basic properties of clinical eye movement recording techniques. In Sanders EACM, de Keizer RJW, Zee DS (eds). Eye movement disorders. Dordrecht: Martinus Nyhoff, 1987, 69-82.

${ }^{10}$ Robinson DA: A method of measuring eye movement using a scleral search coil in a magnetic field. IEEE Trans Biomed Eng 1963, 10: 137-45.

"Dell'Osso LF and Daroff RB: Eye movement characteristics and recording techniques. In Duane TD, Jaeger EA (eds). Clinical Ophthalmology Philadelphia: Harper Row, 1982, (2)9: $1-5$. 